\title{
CRACK PROPAGATION IN RECTANGULAR THIN-WALLED PLATES WITH CONCENTRATORS SUBECTED TO CYCLING LOADING
}

\author{
H. Kopecki, J. Zacharzewski
}

Rzeszów University of Technology, Department of Applied Mechanics and Robotics, Powstancow Warszawy Ave. 8, 35 959 Rzeszow, Poland.E-mail: hkopecki@prz.rzeszow.pl

Received 712 2004, accepted 29082005

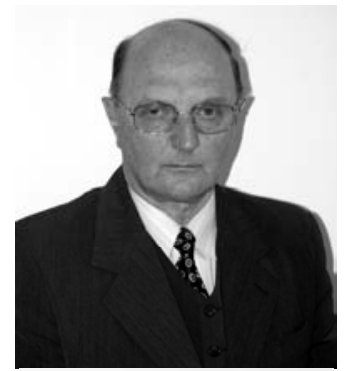

Henryk KOPECKI, D.Sc., Ph.D., Eng., full professor

Rzeszow University of Technology, Faculty of Machine Building and Aviation, Department of Applied Mechanics and Robotics.

Research fields: Mechanics of solids and structure, numerical and experimental analysis of thin-walled structures: static, stability, post buckling states, fracture mechanics.

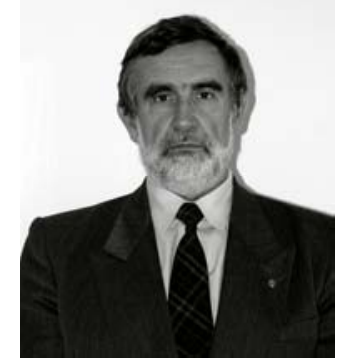

Jan ZACHARZEWSKI, Ph.D., Eng., assistant professor

Rzeszow University of Technology, Faculty of Machine Building and Aviation, Department of Applied Mechanics and Robotics.

Research fields: Numerical and experimental analysis of thin-walled structures: static, buckling, and post buckling analysis, fracture mechanics, fatigue analysis.

Abstract. The paper presents the results of experimental investigations of the crack propagation process in thin-walled elements made from optically active materials (polycarbonate), making the visualization of the field possible isochromatics at the front of the crack. Influence of the orientation of principal directions was analyses An experimental investigation of thin-walled plate with circular and rectangular cutouts subjected to cycling shear loading was made. Using the photo-stress method, propagation of the crack and the field of isochromatics were recorded over time. In parallel, the areas of initiation of fatigue fractures were identified. The results presented in the paper constitute a fragment of a larger process of investigation of thin-walled structures at the stress pattern in conditions of fatigue crack propagation to failure.

Keywords: fracture mechanics, crack propagation, fatigue, photo- stress, numerical analysis.

\section{Numerical and experimental analysis of stress field on crack front zones}

Properties determined on the basis of the experiment of the material are determining the form of constitutive equation.

In accordance to the above-mentioned assertion, properties of the material and size of the plastic zone in proximity of the crack are determining the criterion of the option of accepted constitutive equation $[4,9]$.

Processes of brittle fracture with taking linear and nonlinear equations of the theory of elasticity into consideration were being described in the few papers $[9$, 13]. In case of polymers an application of viscoelastic equations is necessary.

Using the same assumptions as above, it is possible to consider the problem in such cases when the plastic area of deformations is not large. Generally, we apply the theory of plasticity if the stress field on the crack front needs to be determined or if the plastic areas are too large. Magnitudes of plastic areas can be determined using either numerical or experimental method (for example the photo elastic method).

\section{Photo-elastic investigation}

In order to investigate the influence of the position of the crack on the stress field around the crack front, a series of specimens made from epoxy resin material were prepared, in which the no dimensional cracks (zero thickness) were executed in neutral state for: $\beta=90^{\circ}, 75^{\circ}, 60^{\circ}, 45^{0}, 30^{\circ}, 15^{0}, 0^{\circ}$ (Fig 1).

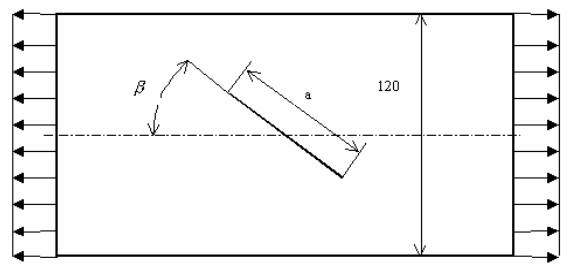

Fig 1. Specimen scheme 
Photo-stress method was used. The results of investigation are presented in Fig 2.

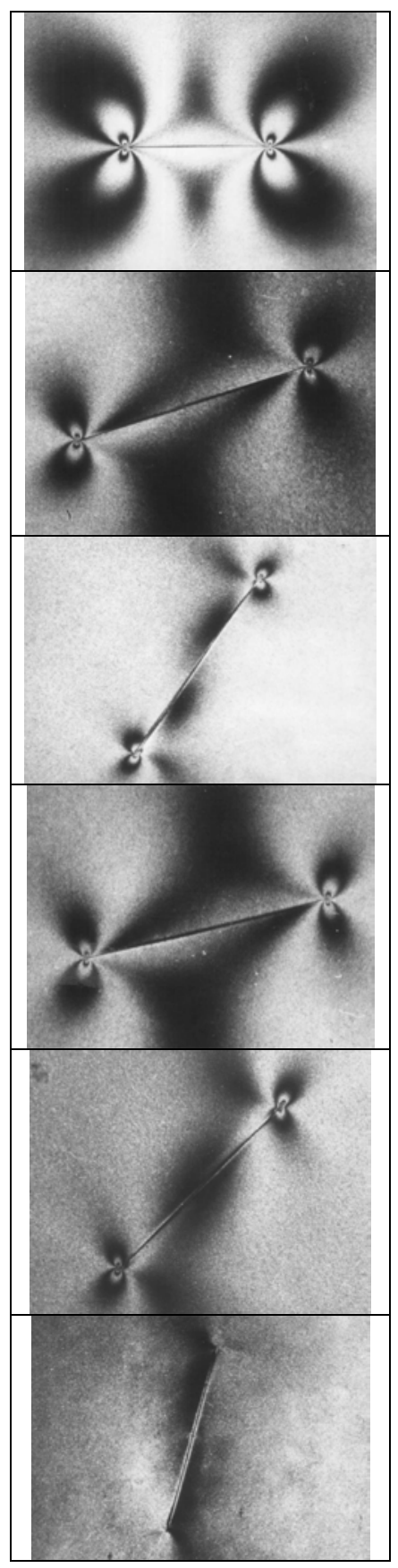

Fig 2. Photographs of isochromatics for six values of angle $\beta$

The relationship between maximum orders of isochromatics and the crack slope angle is presented in Fig 3.

We can present the fundamental equation of photoelasticity for this problem in this form [9]:

$$
\begin{gathered}
m \cdot K_{m}=\sigma_{1}-\sigma_{2}=2 \cdot \tau_{\text {max }} \\
\tau_{\text {max }}=0.5 \cdot K_{m} \cdot m[\mathrm{MPa}]
\end{gathered}
$$

where: $\mathrm{K}_{\mathrm{m}}$ is the elastooptical model constant. In our case, $\mathrm{K}_{\mathrm{m}}=11.75 \mathrm{Mpa}$. Therefore, we can calculate

$$
\tau_{\max }=5.875 \cdot 0.5 \cdot m[\mathrm{MPa}]
$$

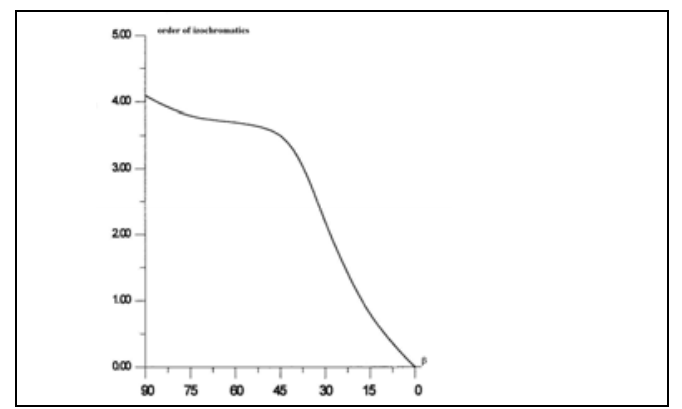

Fig 3. Maximum order of isochromatics versus crack slope angle $\beta$

The relationship between $\tau_{\max }$ in the neighbourhood of the crack front and angle of slope $\beta$ is presented in Fig 4.

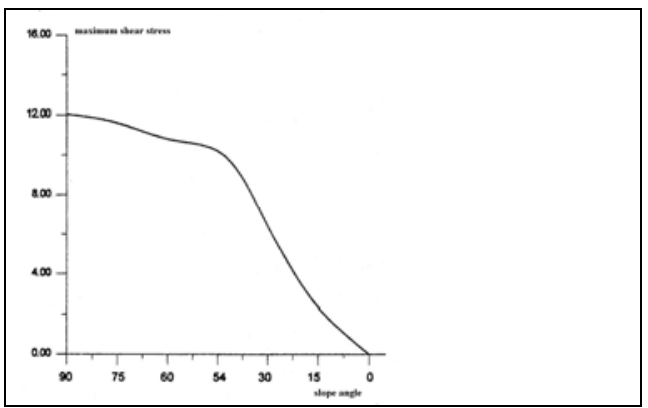

Fig 4. Relationship between $\tau_{\max }$ and angle of slope

\section{Numerical procedure}

According to the theory of elasticity the stress components on the crack front can be determined as follow [9]:

$$
\sigma_{x}=\frac{K_{1}}{\sqrt{2 \pi r}} \cos \frac{\Theta}{2}\left(1-\sin \frac{\Theta}{2} \sin \frac{3}{2} \Theta\right)
$$

$$
\tau_{x y}=\frac{K_{1}}{\sqrt{2 \pi r}} \cos \frac{\Theta}{2}\left(\sin \frac{\Theta}{2} \cos \frac{3}{2} \Theta\right)
$$

$$
\sigma_{y}=\frac{K_{1}}{\sqrt{2 \pi r}} \cos \frac{\Theta}{2}\left(1+\sin \frac{\Theta}{2} \sin \frac{3}{2} \Theta\right)
$$

and displacements

$$
\mathrm{U}=\frac{K_{1}}{2 v} \sqrt{\frac{r}{2 \pi}} \cos \frac{\Theta}{2}\left(\chi-1+2 \sin ^{2} \frac{\Theta}{2}\right)
$$




$$
\mathrm{V}=\frac{K_{1}}{2 v} \sqrt{\frac{r}{2 \pi}} \sin \frac{\Theta}{2}\left(\chi+1-2 \cos ^{2} \frac{\Theta}{2}\right)
$$

For the specimen shown on the Fig 1.

$$
\begin{array}{r}
\alpha=\frac{1-\frac{a}{2 w}+0.362 \frac{a^{2}}{w^{2}}}{\sqrt{1-\frac{a}{w}}}=1.014 \\
K_{1}=0.871 \sqrt{m},
\end{array}
$$

$$
\sigma=\frac{P}{A}=3.17[\mathrm{MPa}]
$$

where:

$$
\chi=\frac{3-v}{1+v}, \text { and } V \text { - Poisson ratio. }
$$

The stress intensity factor for the structure with slope crack is presented in the form:

$$
K_{1}=\alpha \cdot \sigma \sqrt{\pi \cdot a}(4)
$$

In accordance to above-mentioned dependence contour lines of the stress state components, for the angle of slope $\beta=90^{\circ}$ were presented in figures $5-7$.

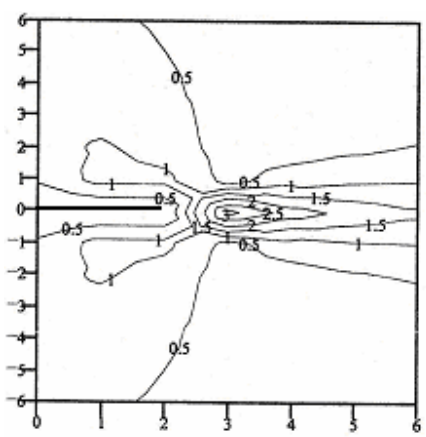

Fig 5. $\sigma_{\mathbf{x}}-$ contour lines

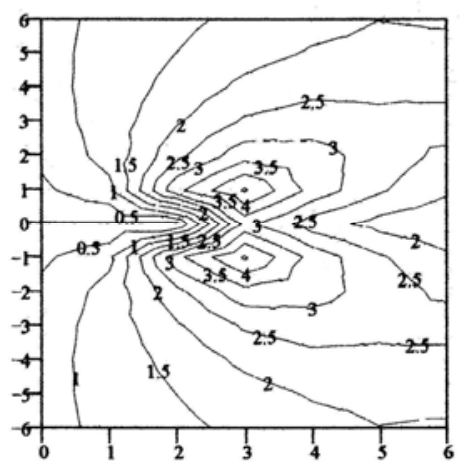

Fig 6. $\sigma_{\mathrm{y}}-$ contour lines

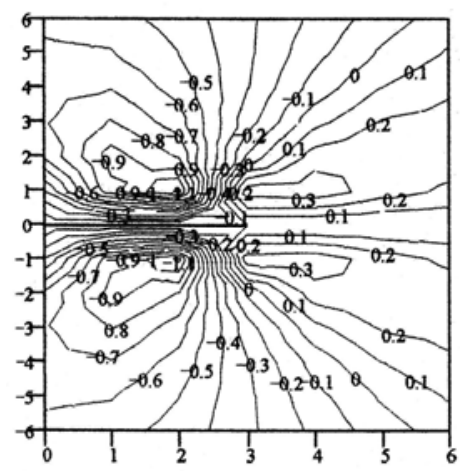

Fig 7. $\tau_{\mathrm{xy}}-$ contour lines

The $\tau_{\max }$ component is calculated according to formula (9),

$$
\tau_{\max }=\frac{1}{2}\left(\sigma_{1}-\sigma_{2}\right)=\sqrt{\frac{\left(\sigma_{x}-\sigma_{y}\right)^{2}}{2}+\tau_{x y}{ }^{2}}
$$

and stress distribution is presented in Fig 8.

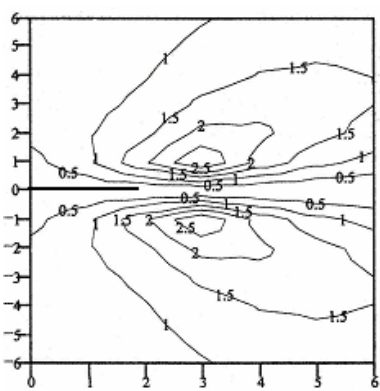

Fig 8. $\tau_{\max }-$ distribution

Comparing results of the experiment and numerical counts it is necessary to find existence full similarities in received of stress pattern. Quantitative dissimilarities in maximum values $(12 \mathrm{MPa}$ - experiment, $3 \mathrm{MPa}$ numerical calculation) can be explained with existence of the singularity on the crack front.

Increasing of accuracy of numerical calculation is possible to receive reducing the analyzed area in proximity of the crack front (Fig 9).

Effective stresses pattern in proximity of the crack front are presented on the Fig 10.

Above-mentioned results were received using the ADINA program.

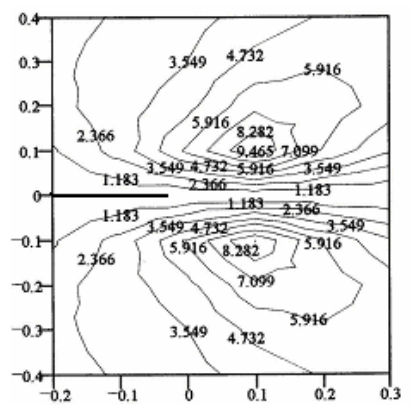

Fig 9. Shear stress pattern in proximity of crack front in the reduced area of calculation 
These results were obtained by FEM ADINA program [1]. Distribution of effective stress in the neighborhood of front crack is presented in Fig 10.

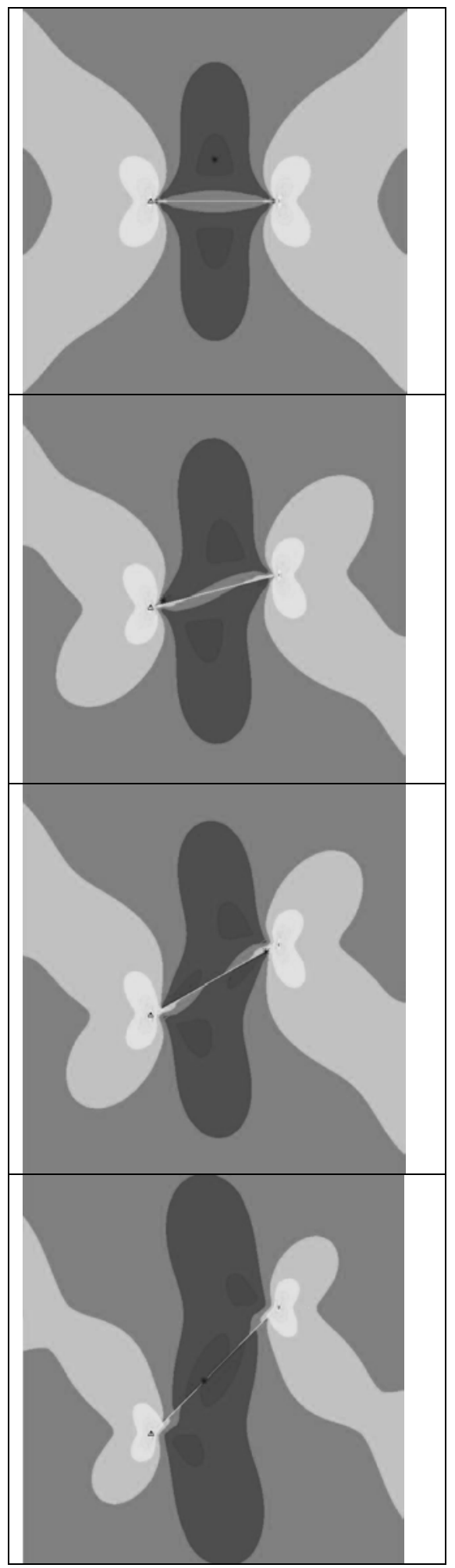

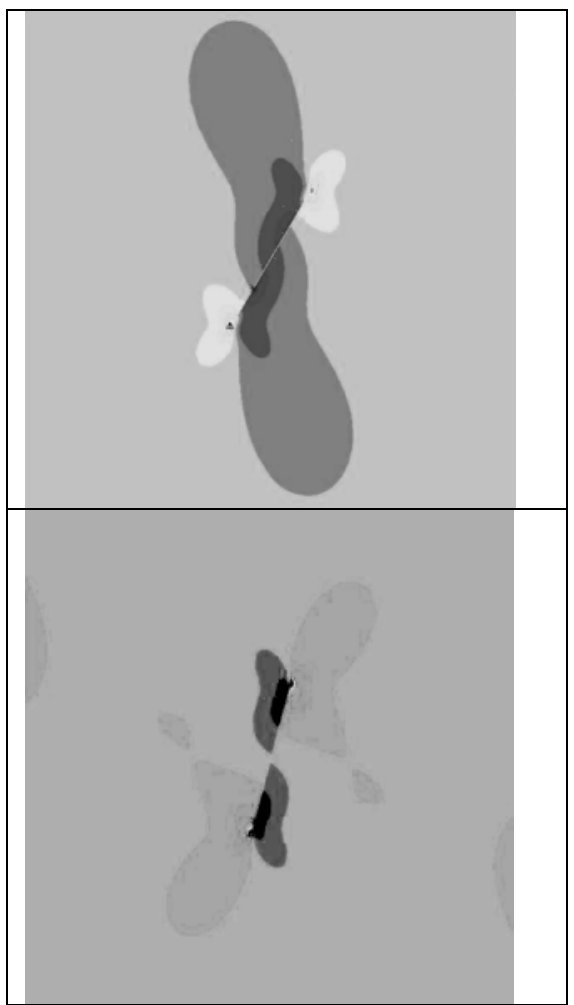

Fig 10. Effective stress pattern in proximity of crack front obtained by ADINA program

\section{Fatigue tests of rectangular plate with cut- out subjected to cycling shear loading}

The durability and operational reliability of carrying structures are conditioned by the character and quantity the load, kind of material and geometry of structures.

Tendencies to increase fatigue durability of design are persuading designers to search for new solutions, where higher durability constitutes the results of geometrically optimizations.

Taking into consideration of arising of the crack in the design is the expensive and labor-consuming process in the analysis of the durability.

Searching for the time of the life of the structures at the stage of designing is returning designers' remark on the chance to predict fatigue failure on the basis of model examinations.

Applying plastics, in particular optically active materials about programmed mechanical properties is creating basis to tests, on the basis of which there is a possibility to estimate damage.

During the tests, we observe the isochromatic field growing in the process by crack, until it reaches critical size.

The problem will be illustrated on a sample of rectangular plate with a cutout in the form of square. The plate is made with polycarbonate. During the test, the structure was subjected to changing load in time. 


\section{Experimental research}

The object of investigation presents the tracing of crack propagation process in time, from the existing moment to the attainment of critical size, for which loss of load capacity came into existence.

Geometry, method of fixing, and loading of plate is presented in Fig 11.

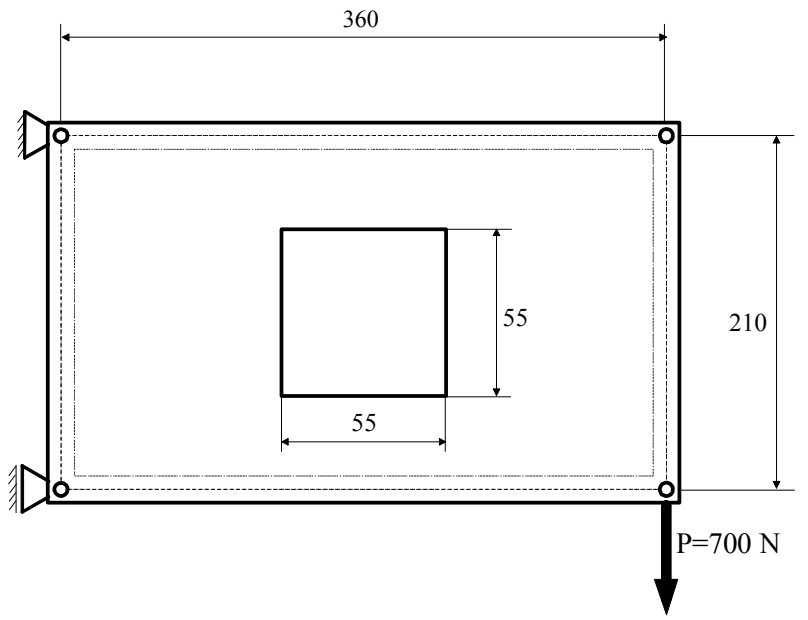

Fig 11. Scheme of loading structure

As we mentioned earlier, the plate was made from polycarbonate, the physical characteristic of which is presented in Fig 12.

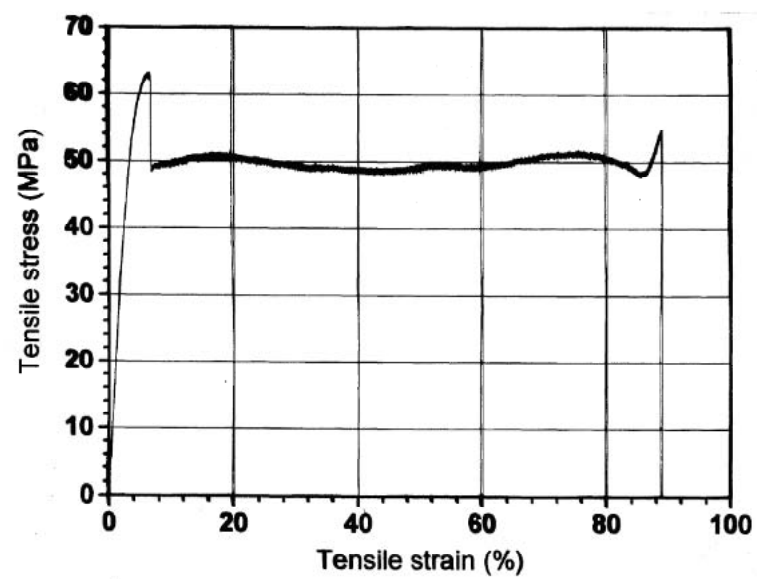

Fig 12. Instantaneous characteristics of polycarbonate

Taking into account the optical active properties of the polycarbonate. Fringe order of isochromatics was being registered in proximity of the front of the promoting crack.

The plate was being loaded with the force pulsating about the fixed average value during examinations. General sight of the research stand is presented in Fig 13.

The stand consists of a few parts: hydraulic feeder with mechanical loading system, electronic control system of the level of the load and frame to fix the plate. The stand is completed by reflection Polaris cop. During the test, the tested plate was covered unilaterally with the reflection varnish making the observation and registration of the field isochromatics possible.
The results of the optical effect are presented in Fig 14 (a-f).

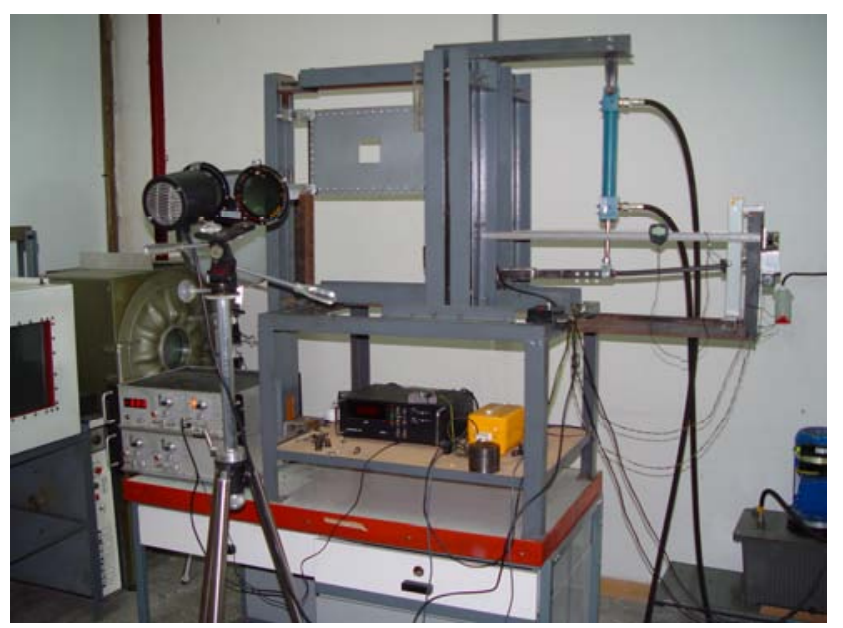

Fig 13. Station for the fatigue test

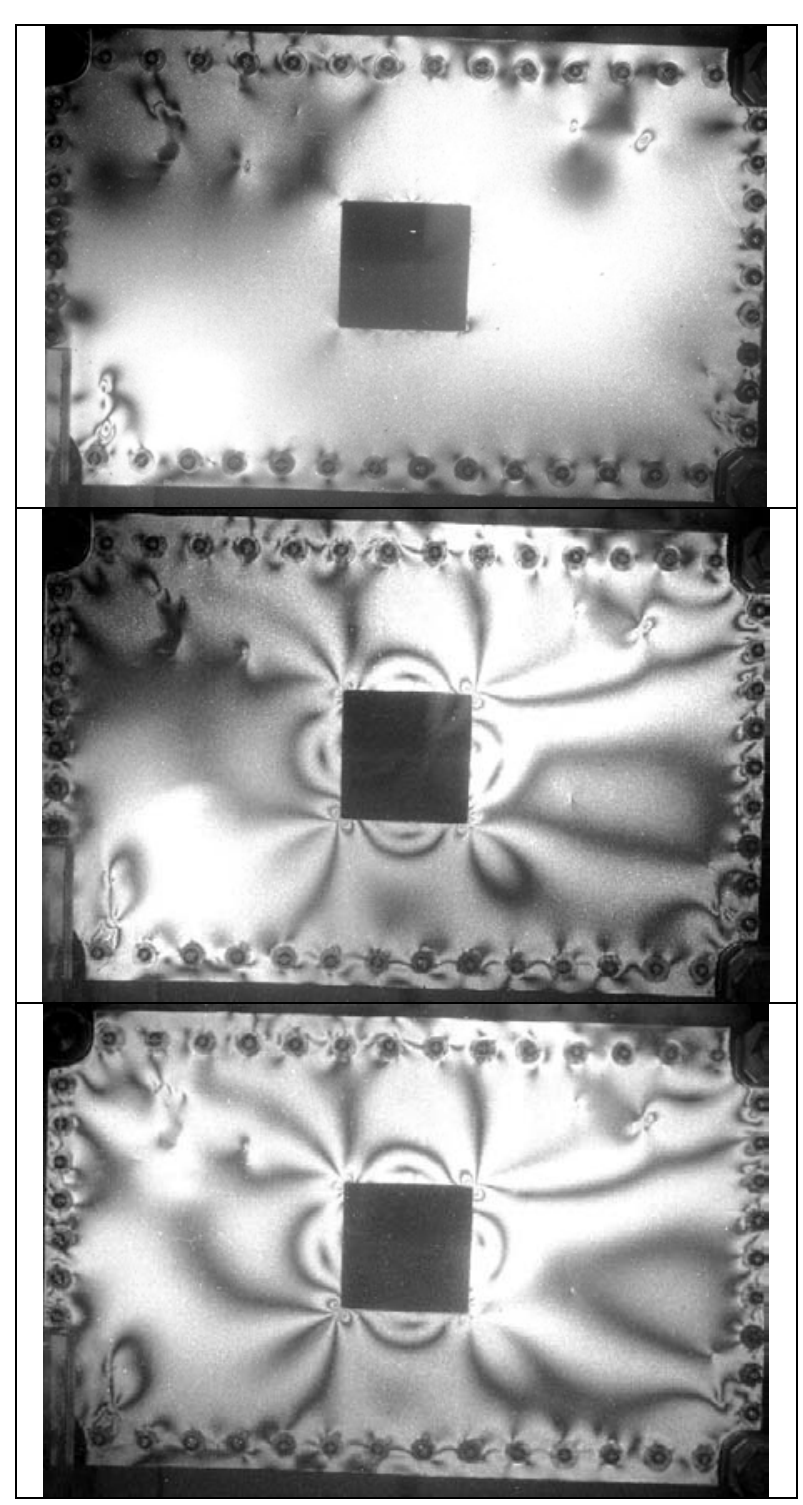




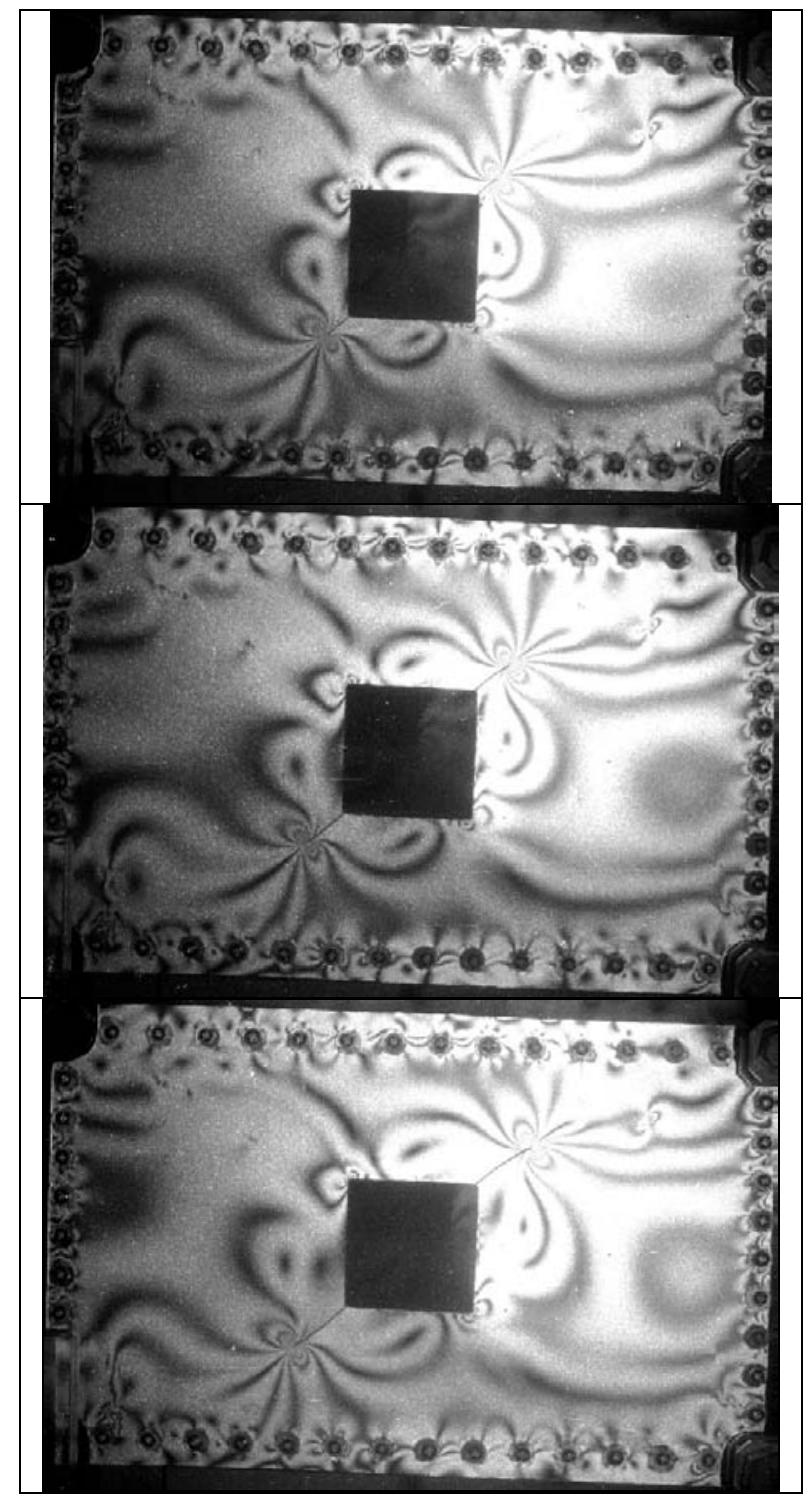

Fig 14. Fields of isochromatics during crack propagation

In Fig 15, crack size dependence in the function of number of cycles is presented.

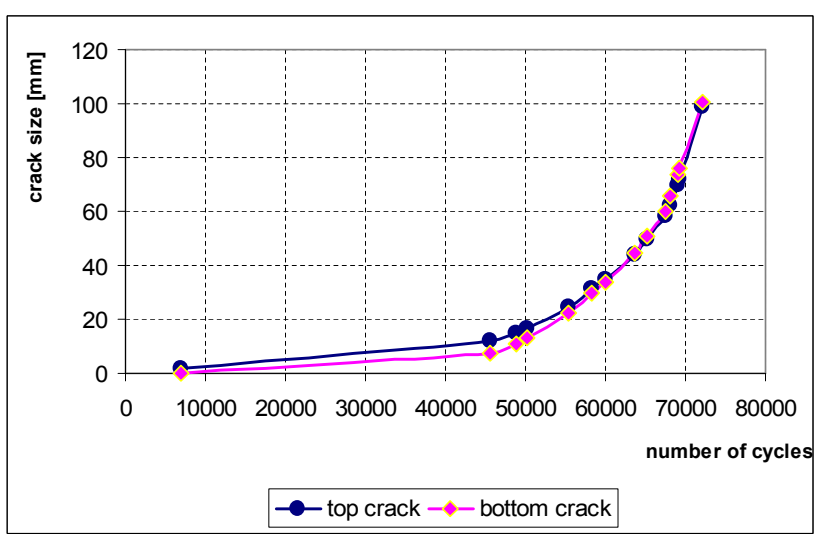

Fig 15. Crack size versus cycle numbers

\section{Conclusions}

On the basis of experimental and numerical results, we are able to formulate the following conclusion:

- using photo-elastic model investigations, the stress field on the front of crack can be determined with regard to qualitative and quantitative analysis. This fact looks to be a very useful during the selection of the constitutive equation applied to counts of the critical length of the crack and the time of life of the structure.

As the result, obtaining of compatibility of effects of the experiment from numerical analysis confirm the purposefulness of numerical analysis supported by experimental research, and photo-mechanics in particular.

\section{References}

1. Adina Theory and Modelling Guide. - Adina R\&D Inc., 2000.

2. Doll W. Fractography and failure mechanisms of polymers and composites. - Elsevier, London, 1989.

3. Hertzberg R.W., Manson J.A. Fatigue of engineering plastics. - Academic Press Inc., 1980.

4. Kopecki H., Zacharzewski J. The crack propagation of thin-walled plates made from phototheological material // $17^{\text {th }}$ Danube-Adria Symposium. - Praha, 2000.

5. Kushenko V.S., Tamusz V.P. Fracture mechanisms of polymers and composites. - Elsevier, London, 1989.

6. Lemaitre J. A Continuous damage mechanics model for ductile fracture // J. Eng. Mater. Technol. 1985. - Vol 107.

7. Lemaitre J. Micro-mechanics of crack initiation // Int. J. Fract. - 1990. - Vol 42.

8. Li X., Hristov., Yee A.F. Influence of cyclic fatigue on the mechanical properties of amorphous polycarbonate // Polymer. - 1995. - Vol 36.

9. Neimitz A. Mechanika pękania. - PWN Warszawa, 1998.

10. Sih G.C., Chao C.K. Fatigue initiation in unmatched specimens subjected to monotonic and cyclic loading // J. Theor. Appl. Fract. Mech. - 1984. - Vol 2.

11. Sih G.C., Tzou D.Y. Crack extension resistance of polycarbonate material // Ibid.

12. Wang B., Lu H., Tan G., Chen W. Strength of damage polycarbonate after fatigue // Theor. Appl. Fract. Mech. - 2003. - Vol 39.

13. Wnuk M.P. Fundamental concepts of damage tolerant design. - University of WisconsinMilwaukee. 